Check for updates

Cite this: Chem. Sci., 2018, 9, 8382

๑ All publication charges for this article have been paid for by the Royal Society of Chemistry

Received 27th June 2018

Accepted 2nd September 2018

DOI: $10.1039 / \mathrm{c} 8 \mathrm{sc} 02842 \mathrm{~g}$

rsc.li/chemical-science

\title{
Covalent organic nanosheets for bioimaging $\dagger$
}

\author{
Gobinda Das, $\ddagger^{\mathrm{a}}$ Farah Benyettou, $\neq^{\mathrm{a}}$ Sudhir Kumar Sharama, ${ }^{\mathrm{b}}$ \\ Thirumurugan Prakasam, ${ }^{a}$ Felipe Gándara, (D) ' Victor A. de la Peña-O'Shea, (D) d \\ Na'il Saleh, (D) e Renu Pasricha, ${ }^{a}$ Ramesh Jagannathan, ${ }^{b}$ Mark A. Olson (iD ${ }^{f}$ \\ and Ali Trabolsi (D) *a
}

Covalent organic nanosheets (CONs) have attracted much attention because of their excellent physical, chemical, electronic, and optical properties. Although covalent organic nanosheets have widely been used in many applications, there are only a few CONs that have been tested for bio-medical applications. Nanometer sized triazine-based nanosheets were obtained by exfoliating their bulk counterparts in water. The obtained nanosheets were dispersible and stable in water with enhanced photoluminescence properties compared to the bulk material. The nanosheets were biocompatible and non-toxic and showed ability to stain HeLa cell nuclei without additional assistance of an external targeting agent.

\section{Introduction}

Covalent organic nanosheets (CONs) obtained by exfoliation of their counterparts, such as covalent organic frameworks (COFs), ${ }^{1}$ have emerged as a new class of porous, thin twodimensional (2D) nanostructures with distinctive dimension related properties that differ from their corresponding bulk materials. ${ }^{2-6}$ Exfoliation of COFs into CONs reduces the size of the material while also imparting novel physical and chemical properties, which for example, allows for the circumvention of a few serious COF drawbacks, namely their dispersibility and bioavailability within the cells. In this regard, CONs are a promising nanomaterial for applications in biomedicine including drug delivery and bioimaging. .,3,7,8 $^{2}$

Non-toxic CONs with high photoluminescence quantum yields can be of great interest for bioimaging as they are stable in solution, possess tunable optical characteristics, and exhibit limited photobleaching. Reported materials for bioimaging are mainly (i) inorganic in nature with the presence of heavy

${ }^{a}$ Chemistry Program, New York University Abu Dhabi (NYUAD), United Arab Emirates. E-mail: at105@nyu.edu

${ }^{b}$ Engineering Division, New York University Abu Dhabi (NYUAD), United Arab Emirates ${ }^{c}$ The Materials Science Factory, Instituto de Ciencia de Materiales de Madrid - CSIC, Sor Juana Inés de la Cruz 3, 28049 Madrid, Spain

${ }^{d}$ Photoactivated Processes Unit, IMDEA Energy, Avd. Juan de la Sagra 3, Mostoles 28935, Madrid, Spain

${ }^{e}$ United Arab Emirates University, College of Science, Department of Chemistry, Al-Ain, United Arab Emirates

${ }^{f}$ School of Pharmaceutical Science and Technology, Health Science Platform, Tianjin University, 92 Weijin Rd. Nankai, District, Tianjin 300072, P. R. China

$\dagger$ Electronic supplementary information (ESI) available. See DOI: $10.1039 / \mathrm{c} 8 \mathrm{sc} 02842 \mathrm{~g}$

\$ These authors contributed equally to this work. metals, ${ }^{9}$ (ii) carbon nitride, ${ }^{\mathbf{1 0}}$ or (iii) graphene-based nanomaterials, ${ }^{\mathbf{1 1 - 1 3}}$ all with considerably challenging syntheses. ${ }^{\mathbf{1 4}}$ While several examples in the literature have employed CONs in drug delivery, ${ }^{2,15}$ they have yet to be investigated for bioimaging.

We describe here the synthesis of a crystalline, porous, and luminescent triazine-based COF (denoted as TTA-DFP COF) from the condensation of 2,6-diformylpyridine (DFP) with $4,4^{\prime}, 4^{\prime \prime}$-(1,3,5-triazine-2,4,6-triyl)trianiline (TTA) under $30 \mathrm{~min}$ of microwave irradiation (Fig. 1). As synthesized, the bulk material is constituted of nanosheets and is weakly fluorescent with micrometer lateral dimensions. Exfoliation of the bulk material in pure water drastically reduces the lateral dimensions to the nanometer scale and enhances the photoluminescence properties by a factor of 6 . The TTA-DFP CONs exhibit a high luminescence efficiency with a maximum peak centred at $435 \mathrm{~nm}$ when excited at $\lambda_{\max }=375 \mathrm{~nm}$.

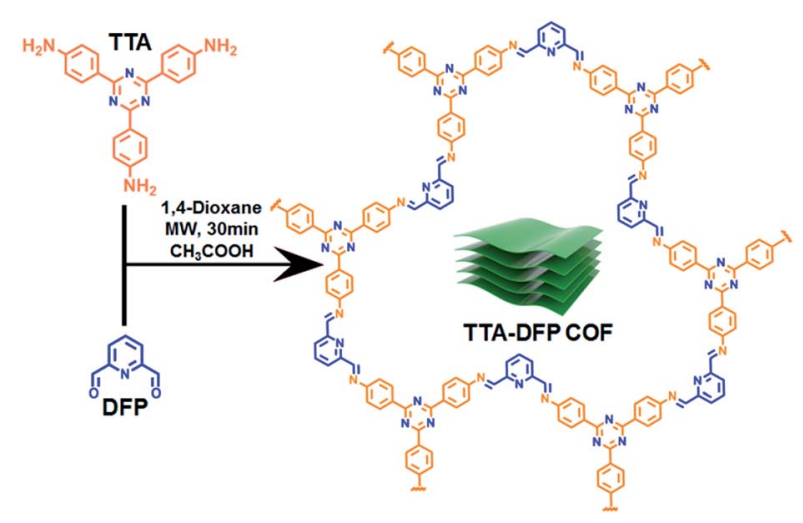

Fig. 1 Chemical structure and synthetic route of the TTA-DFP COF under microwave irradiation. Cartoon representation (green block) illustrates the stacked nanosheets of the TTA-DFP COF. 
Cellular labelling is of great interest in biology, in particular the nucleus, which is regarded as one of the main targets for cancer therapeutics. ${ }^{16}$ Nuclear staining dyes (organic or peptidebased) are limited by poor photo-sensitivity, challenging syntheses, and cost. ${ }^{11}$ Incubation experiments with HeLa cells demonstrated that TTA-DFP CONs could be used for label-free selective intracellular localization within the cellular nucleus without hampering the cell architecture and viability, thus making this intrinsically luminescent CON material a promising nuclear probe for bioimaging.

\section{Results and discussion}

The bulk material, denoted as TTA-DFP COF, was synthesized by co-condensation of DFP (12 mg, $0.09 \mathrm{mmol}, 3$ equiv.) and TTA (21.24 mg, $0.06 \mathrm{mmol}, 2$ equiv.) in $3 \mathrm{~mL}$ of anhydrous 1,4-dioxane in the presence of a catalytic amount of acetic acid, sealed in a $10 \mathrm{~mL}$ glass microwave tube and heated under microwave irradiation at $110{ }^{\circ} \mathrm{C}$ for $30 \mathrm{~min}$ (Fig. 1). The formed yellowish precipitate was collected by filtration, washed with 1,4-dioxane and ethanol, and dried at $110{ }^{\circ} \mathrm{C}$ for $12 \mathrm{~h}$. After centrifugation and multiple washings with ethanol, the product was completely insoluble in water and all organic solvents tested, including THF, acetone, $\mathrm{CHCl}_{3}$, DMF and DMA, indicating the formation of a stable polymeric material.

The formation of imine $(\mathrm{C}=\mathrm{N})$ linkages for the as-synthesized material was first confirmed by Fourier-transform infrared (FT-IR) spectroscopy. The material displayed stretching modes at $1625 \mathrm{~cm}^{-1}$ as well as the disappearance of the TTA primary amine stretches at $\sim 3420 \mathrm{~cm}^{-1}$ and the DFP aldehyde stretch at $1700 \mathrm{~cm}^{-1}$ (Fig. S1†). Thermogravimetric analyses (TGA) revealed that the TTA-DFP COF exhibits excellent thermal stability, maintaining more than $60 \%$ of its weight up to $500{ }^{\circ} \mathrm{C}$ (Fig. S2†).

The crystallinity of the as-synthesized material was demonstrated by powder X-ray diffraction (PXRD), which shows a strong peak at $2 \theta=5^{\circ}$ that can be assigned to the (110) plane of the regularly ordered lattice, as well as a broad peak centred at $2 \theta=25.6^{\circ}$, corresponding to the reflection from the (003) plane (Fig. 2a).
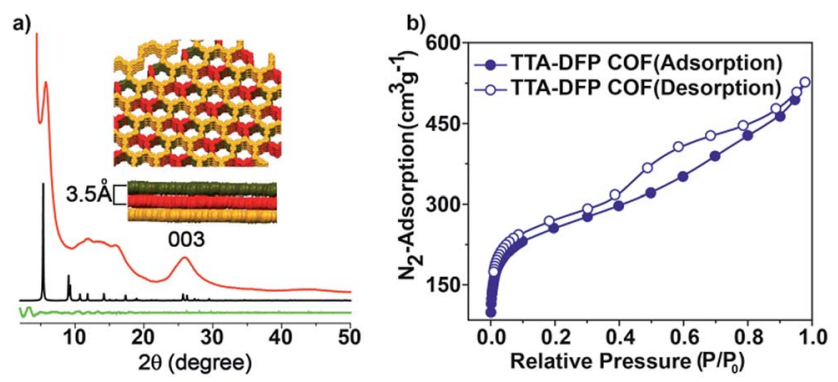

Fig. 2 (a) PXRD patterns and simulated structures of the TTA-DFP COF: experimental (red line), simulated (black line) and difference (green line). TOP view: space-filling representation of the stacked layers of the TTA-DFP COF. The simulated unit cell parameters are $a=$ $b=33.0 \AA$ and $c=10.39 \AA$. (b) Nitrogen adsorption/desorption isotherms at $77 \mathrm{~K}$ of the TTA-DFP COF.
The spacing between the $\pi-\pi$ stacking planes was calculated and found to be $\sim 3.5 \AA$, which matches the simulated structure. Following reticular chemistry principles, crystal structure models were built (Fig. 2a) based on the geometry of the building blocks. Models based on distorted hcb layered structures were generated in the trigonal $P 3$ space group and geometrically optimized with universal force field energy minimizations. The best agreements between experimental and simulated PXRD patterns were obtained for layer stacked structures with an $\mathrm{ABC}$ sequence, resulting in unit cell parameters of $a=b=33.0 \AA$ and $c=10.39 \AA\left(R_{\mathrm{wp}}=2.07 \%\right.$ and $R_{\mathrm{p}}=$ $0.76 \%$ after Pawley refinement). The structure is distorted compared to regular hcb layers because the meta disposition of the aldehyde groups in the pyridine linker imposes a non-linear connection between the triangular nodes, which is, indeed, not common in reported COFs. On account of the sheet-like layered morphology of TTA-DFP COFs having an imperfect long-range order, broadening of the wide-angle diffraction peaks was observed. PXRD patterns of this sort are commonly reported for other layered triazine-based covalent organic networks. ${ }^{17-20}$

The porosity of the TTA-DFP COF was evaluated by measuring nitrogen gas adsorption/desorption isotherms at $77 \mathrm{~K}$ (Fig. 2b). The samples were activated at $85{ }^{\circ} \mathrm{C}$ for 24 hours prior to the measurements in order to remove solvent and trapped gas. The TTA-DFP COF exhibits a sorption isotherm, which is a characteristic of microporous materials. ${ }^{21}$ The Brunauer-Emmett-Teller (BET) surface area of the TTA-DFP COF was found to be $900 \mathrm{~m}^{2} \mathrm{~g}^{-1}$, while the pore size distribution of the COF was calculated with a DFT fitting of the adsorption branch, based on a model with cylindrical pores. A narrow distribution of pores with an average width of $1.7 \mathrm{~nm}$ (Fig. S3†) was obtained which is in agreement with the proposed structural models, where hexagonal channels are formed upon stacking of the layers. The calculated pore volume for the TTADFP COF is $0.76 \mathrm{~cm}^{3} \mathrm{~g}^{-1}$.

The morphology of the TTA-DFP COF was investigated by high resolution transmission electron microscopy (HRTEM) and atomic force microscopy (AFM). HRTEM images showed transparent nanosheets, an indication of the thickness of TTADFP COFs (Fig. 3a). AFM analysis of TTA-DFP COFs revealed a 2D sheet-like morphology (Fig. 3b). The topological height profile for the $2 \mathrm{D}$ sheets was found to be $1.5 \mathrm{~nm}$. PXRD analysis indicates an interlayer stacking distance of $0.35 \mathrm{~nm}$, which is consistent with the well-defined lattice fringes obtained from HRTEM analysis (0.38 nm, Fig. 3a). Based on these microscopy data, we determined that TTA-DFP COFs are comprised of about four stacked layers.

Prior to their use for biomedical applications, it is important to process these materials to nanometer sizes while also imparting water solubility and stability. ${ }^{22}$ For these reasons, liquid exfoliation processing (LEP) ${ }^{23-27}$ from bulk to submicronsized TTA-DFP CONs in water was performed using ultrasonication for 5 hours (Fig. 3, top). Exfoliation was confirmed by TEM imaging of the sample which was comprised of large quantities of $2 \mathrm{D}$-nanosheets with an average size of $\approx 22 \mathrm{~nm}$ (Fig. 3c, S4 and S5†). The clear distinct Tyndall effect of TTADFP CON solution (inset Fig. 3c) also indicates the presence of 

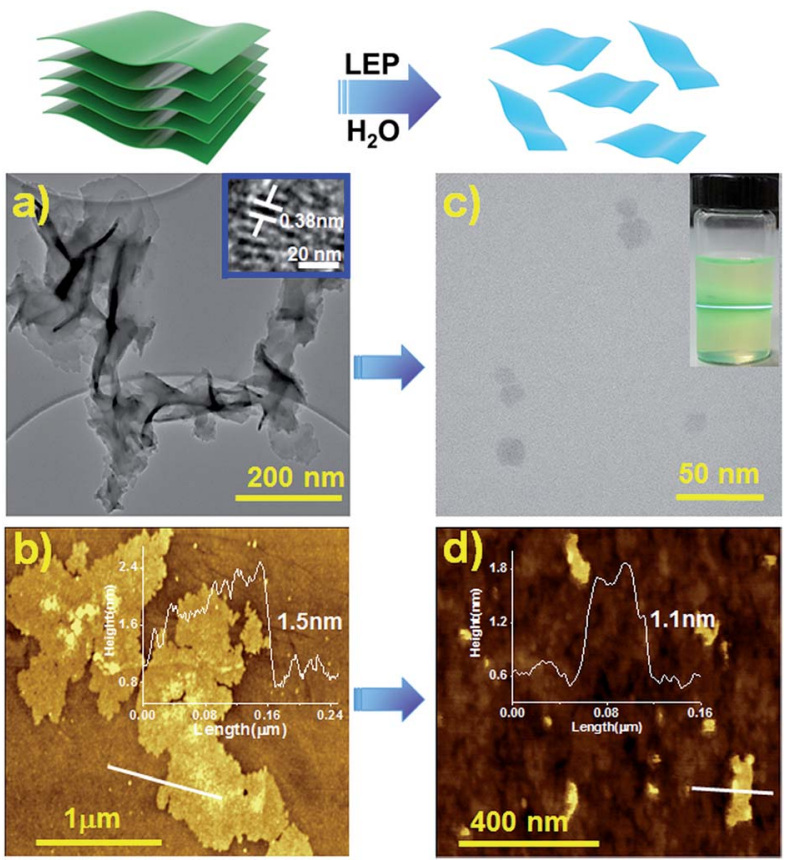

Fig. 3 Schematic representation of the liquid exfoliation process (LEP) in water ( 5 hours). HR-TEM ( $a$ and $c$ ) and AFM ( $b$ and $d$ ) images with line profiles of the TTA-DFP COF and TTA-DFP CONs, respectively. Inset of (c) shows the Tyndall effect of TTA-DFP CONs in water.

highly monodisperse ultrathin nanosheets in water. ${ }^{\mathbf{1 0}}$ AFM confirmed the formation of ultrathin 2D-nanosheets with smaller lateral diameters (Fig. 3d). Upon liquid phase exfoliation, the sheet thickness decreased from 1.5 to $1.0-1.3 \mathrm{~nm}$ (Fig. 3d). Not only did the diameter of the nanosheets decrease but also sheets of uniform thickness and shape were produced. PXRD analysis showed that the intensity of the (110) peak gets reduced upon exfoliation and the (003) peak gets broader (Fig. S7†), which is consistent with the smaller number of vertically stacked layers. Such peak broadening is also noted for previously reported CONs. ${ }^{28}$ Aqueous solutions of exfoliated nanosheets were found to be stable with excellent dispersibility with no precipitation observed over time. At $\mathrm{pH} 7.4$ in water, the hydrodynamic diameter of TTA-DFP CONs was found to be $37.8 \mathrm{~nm}$ (Fig. S8†). The stability of TTA-DFP CONs was then investigated in foetal bovine serum (FBS) to simulate the physiological conditions in cells (Fig. S9†). The hydrodynamic diameter of the TTA-DFP CONs was found to be $23.55 \mathrm{~nm}$, which is close to the size observed by TEM $(22 \mathrm{~nm})$. Additionally, when the mixture was stirred for twenty-four hours at room temperature, the size remained unchanged $(22.80 \mathrm{~nm})$. Together, these results confirm the stability and minimal aggregation of TTA-DFP CONs under simulated physiological conditions.

The electronic properties of the bulk material and the exfoliated nanosheets were studied by absorption and fluorescence spectroscopies. As shown in Fig. 4a, the UV-vis absorption maximum for an aqueous dispersion of TTA-DFP CONs is blue shifted compared to the bulk material $\left(\lambda_{\max }=387 \mathrm{~nm}\right.$ versus $438 \mathrm{~nm}$, respectively). In water, the TTA-DFP COF exhibited
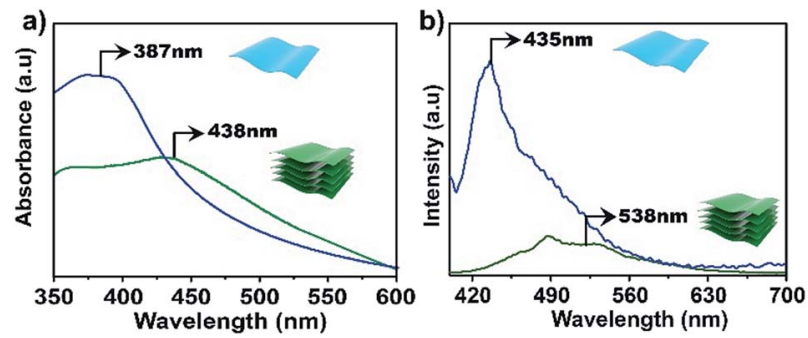

Fig. 4 UV-vis (a) and emission (b) spectra of bulk TTA-DFP COFs (green line) and exfoliated TTA-DFP CONs (blue line) dispersed in water at room temperature, $\lambda_{\mathrm{ex}}=375 \mathrm{~nm}$.

a broad emission band centred at $\lambda_{\max }$ of $538 \mathrm{~nm}$ when excited at $375 \mathrm{~nm}$ (Fig. 4b). After the exfoliation of the bulk material into TTA-DFP CONs, the fluorescence emission spectrum is wider with a maximum that is blue shifted from $538 \mathrm{~nm}$ to $435 \mathrm{~nm}$, with an intensity enhancement by a factor of $\sim 6$ (Fig. 4b), which is the result of reduced interlayer $\pi-\pi$ interactions in the nanosheets. Such a change in the $\pi-\pi$ interactions induces a sharp increase of the optical band gap, from $2.45 \mathrm{eV}$ in the bulk sample to $2.7 \mathrm{eV}$ in the nanosheets and explains the observed increase in intensity. ${ }^{\mathbf{4 , 5 , 1 0 , 2 9 - 3 1}}$ The photoluminescent nature of both the TTA-DFP COF and TTA-DFP CONs can be explained by intramolecular donor/acceptor charge transfer from the phenyl groups (donors) to the central triazine moieties (acceptors). A control experiment, in which the luminescence of the TTA monomer condensed with pyridinemonoaldehyde was measured, revealed that the monomer was not luminescent in water (Fig. S10 $\dagger$ ). The less emissive character of the bulk material could be explained by the aggregationinduced quenching mechanism resulting from the $\pi-\pi$ layered structure of the COF. ${ }^{21}$

To gain more insight into the band structure of the TTA-DFP COF, plane wave density functional theory (PW-DFT) calculations were performed for bulk and single layers following optimization of the calculated crystal structures to determine their electronic and optical properties, and differences between bulk and single layers. The density of states (DOS) shows (Fig. 5) an increase of states at the edge of the conduction band in the bulk sample, indicating a blue shift in both the wavelengths of light absorbed and subsequent emission. As expected, the TTADFP COF displays semiconducting behaviour, with a Valence Band (VB) and a Conduction Band (CB) formed by hybridized $\mathrm{C}$ $2 \mathrm{p}$ and $\mathrm{N} 2 \mathrm{p}$ states as depicted in the charge density plots (Fig. 5). In addition, the energy gaps obtained from HSE06 calculations are $2.45 \mathrm{eV}$ for the bulk sample and $2.7 \mathrm{eV}$ for a single layer nanosheet (Fig. S11 $\dagger$ ). These results are in agreement with the optical measurements obtained from UV-vis diffuse reflectance spectroscopy (UV-vis DRS) which reveal a broad absorption band with an indirect band gap of $2.55 \mathrm{eV}$ (Fig. S12 $\dagger$ ). Time dependent density functional theory (TD-DFT) calculations were used to determine the optoelectronic properties of the materials and indicate that the electronic transitions mainly occur with UV excitation and are associated with intramolecular charge-transfer processes from several highest occupied molecular orbital (HOMO) degenerate orbitals centred 

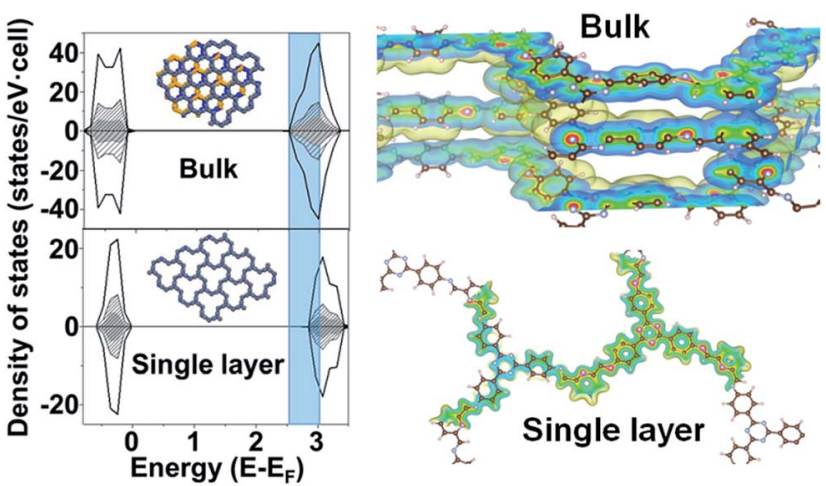

Fig. 5 Total density of states (DOS) (black) and atom-projected density of states (PDOS) for C (black dash) and N (grey) and schematic representation of the isosurfaces of a section of charge density for: bulk and single layer nanosheets. Atom colors: brown for C, blue for $\mathrm{N}$, and white for $\mathrm{H}$. Shadow part clearly indicates an increase of states at the edge of the conduction band in the bulk sample. in the imine moieties to the lowest unoccupied molecular orbital (LUMO) degenerate orbitals (Fig. S13†). Finally, we calculated the surface energy of the monolayer from DFT energy calculations. The calculated surface energy is $98.8 \mathrm{~mJ} \mathrm{~m}^{-2}$, which is comparable to the value reported for other twodimensional structures ${ }^{\mathbf{1 0}}$ and also similar to the surface energy of water $\left(\sim 102 \mathrm{~mJ} \mathrm{~m}^{-2}\right)$ suggesting a potentially high dispersibility of the TTA-DFP COF in this solvent.

The enhanced luminescence properties of the exfoliated TTA-DFP CONs, their nanometer size and their high dispersibility in biological media compelled us to use them as a fluorescent probe to perform cellular labelling on HeLa cells. Cells treated with the exfoliated nanosheets $\left(100 \mu \mathrm{g} \mathrm{mL}^{-1}\right)$ for 30 minutes up to 24 hours exhibited a strong blue signal $\left(\lambda_{\mathrm{ex}}=\right.$ $360 \mathrm{~nm}$ ) which increases in intensity with time demonstrating the ability of the material to internalize and accumulate into cells. Time-dependent incubation experiments (Fig. 6 a-c and $\mathrm{S} 14-\mathrm{S} 17 \dagger)$ revealed that TTA-DFP CONs could enter the cell
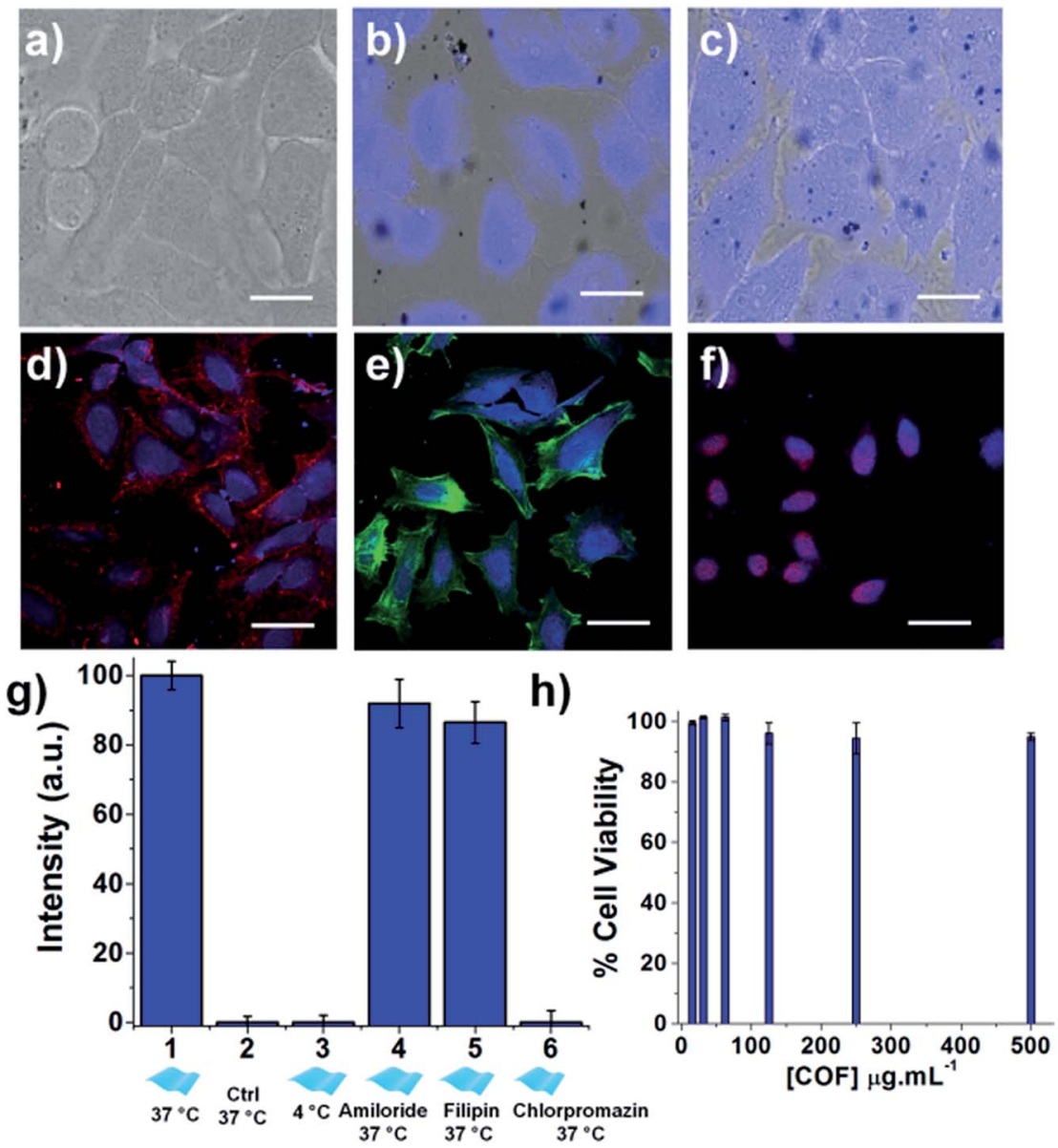

Fig. 6 In vitro study. Overlay of bright field and fluorescence images of HeLa cells incubated with the TTA-DFP CONs (100 $\left.\mu \mathrm{g} \mathrm{mL}{ }^{-1}\right)$ for (a) 0 , (b) 60 and (c) 180 minutes of incubation, showing that accumulation of the TTA-DFP CONs in the nucleus does not hamper the cell integrity ( $\lambda_{\text {ex }}=$ $360 \mathrm{~nm}$; scale bars $=10 \mu \mathrm{m})$. Confocal images of HeLa cells incubated for 4 hours with the exfoliated TTA-DFP CONs (50 $\mu \mathrm{g} \mathrm{mL}{ }^{-1}$ ) and (d) a plasmic membrane marker, (e) an actin marker and (f) a nucleus marker, showing that after internalization in cells, the exfoliated TTA-DFP CONs specifically target the nucleus (blue channel $\left(\lambda_{\mathrm{ex}}=405 \mathrm{~nm}\right)$, red channel $\left(\lambda_{\mathrm{ex}}=633 \mathrm{~nm}\right)$, green channel $\left(\lambda_{\mathrm{ex}}=561 \mathrm{~nm}\right)$, and scale bars $=$ $20 \mu \mathrm{m})$. (g) Quantitative comparison of fluorescence intensity in HeLa cells incubated with TTA-DFP CONs $\left(50 \mu \mathrm{g} \mathrm{mL}{ }^{-1}\right)$ at $4{ }^{\circ} \mathrm{C}$ versus $37^{\circ} \mathrm{C}$ as well as in the presence of endocytotic inhibitors. (h) Viability assessment of HeLa cells after $48 \mathrm{~h}$ incubation with increasing concentrations of exfoliated TTA-DFP CONs up to $500 \mu \mathrm{g} \mathrm{mL}^{-1}$. 
nucleus after $30 \mathrm{~min}$. It is worth noting that TTA-DFP CONs are stable under acidic conditions with no hydrolysis of the imine bond observed. Also, the monomers are not fluorescent. We conclude that the fluorescence observed cannot come from fragmentation of the TTA-DFP CONs but from their presence in the nucleus. Few aggregates can be seen outside cells but are not fluorescent and therefore cannot be considered as aggregated nanosheets but are actually due to a small fraction of nonexfoliated material which is not fluorescent at this excitation wavelength.

To further confirm the localization of the nanosheets in the cells, the nucleus, the membrane, and the cytoplasm of the cells were specifically labelled using organelle markers and colocalized with the exfoliated TTA-DFP CON signal (Fig. 6d-f). The blue emission in the cells arising from the nanosheets does not overlap with the emission of either the membrane (Fig. 6d) or the actin in the cytoplasm (Fig. 6e). However, the blue emission does overlap with the fluorescence staining from the red labelled nucleus (Fig. 6f), with also accumulation in the endoplasmic reticulum (ER). The accumulation of the TTA-DFP CONs in the ER is particularly interesting because the lumen of the ER is contiguous with the space between the inner and outer nuclear membranes. ${ }^{32}$ Recently, Mal et al. ${ }^{6}$ reported the interactions of self-exfoliated ionic covalent organic nanosheets with DNA, which might explain the accumulation of the TTA-DFP CONs into the nucleus.

In order to determine the relative amounts of active versus passive mechanisms involved in TTA-DFP CON internalization, we compared the relative intensity of fluorescence in cells incubated with TTA-DFP CONs for $1 \mathrm{~h}$ at either $4{ }^{\circ} \mathrm{C}$ or $37{ }^{\circ} \mathrm{C}$ (Fig. S21†). The fluorescence intensity in the cells incubated at $4{ }^{\circ} \mathrm{C}$ is considerably diminished compared to the cells' fluorescence intensity at $37{ }^{\circ} \mathrm{C}$, indicating that the active mechanisms of internalization, such as endocytosis, are predominant. To understand the TTA-DFP CON cellular uptake mechanism, HeLa cells were pre-treated with different inhibitors of various endocytotic mechanisms (Fig. S21†). For amiloride and filipintreated cells, the fluorescence intensity did not change much after 1 hour of incubation, suggesting that macropinocytosis and caveolae-mediated endocytosis are not involved in the internalization process. ${ }^{33}$ In contrast, the fluorescence intensity of the chlorpromazine-treated cells was significantly less pronounced, implying that clathrin-mediated endocytosis may be the major pathway for TTA-DFP CON internalization (Fig. 6g). ${ }^{33}$ Clathrin-mediated endocytosis is one of the pathways that sorts molecules to organelles such as the Golgi, the ER and the nucleus. ${ }^{34,35}$ This result is consistent with the observation of TTA-DFP CON accumulation in the ER and nucleus. In order to assess if the intensity of the fluorescence arising from TTA-DFP CONs diminishes over time inside the cells, HeLa cells were incubated with TTA-DFP CONs for $4 \mathrm{~h}$ and then subsequently incubated for $24 \mathrm{~h}$ with fresh media without TTA-DFP CONs (Fig. S18†). Fluorescence images showed that the cells' fluorescence intensity does not fade and remains strong and localized within the nucleus. It is worth noting that after 24 hours of incubation with TTA-DFP CONs, cells were present in the ER and nucleus demonstrating that even after prolonged exposure of cells to TTA-DFP CONs up to 24 hours, the sheets continue accumulating into the cells without hampering the cell integrity. We conclude that after internalization through clathrin-mediated endocytosis within the HeLa cells, the exfoliated TTA-DFP CONs specifically target and label the nucleus in a relatively short time of 30 minutes. A similar phenomenon was observed for graphene quantum dots with a diameter below $100 \mathrm{~nm} \cdot{ }^{13}$

The exfoliated TTA-DFP CONs did not weaken cell activity and the cells maintained their normal morphology up to 24 hours of incubation (Fig. S15†), which encouraged us to assess the biocompatibility of this material. Overall the exfoliated TTA-DFP CONs elicit no cytotoxic effects on HeLa cells after 48 hours of incubation with concentrations up to $500 \mu \mathrm{g} \mathrm{mL}$ (Fig. 6h).

\section{Conclusions}

In summary, we have successfully synthesized a crystalline, porous and luminescent triazine-based COF (denoted as TTADFP COF) using a facile microwave-assisted method. Liquidexfoliation of the bulk TTA-DFP COF in water gave rise to nanometer-sized 2D-nanosheets whose luminescence emission was considerably blue shifted and enhanced compared to the bulk material. Our in vitro results following the incubation of the nanosheets with HeLa cells confirmed that the exfoliated materials did not affect the cell architecture and viability, which constitutes a preliminary demonstration of biocompatibility and nontoxicity. Two commercially available blue-emitting dyes are commonly used in biology to stain DNA. One of these two, DAPI, is used to stain dead cells or cells with compromised membranes and has high toxicity. The second is Hoechst 33258, which is less toxic and suitable for staining both live and dead cells. Hoechst enters the cell by an unmediated diffusion transport mechanism through the cell membrane prior to DNA binding. ${ }^{36,37}$ The exfoliated TTA-DFP CONs function as nuclear probes for bioimaging without the additional assistance of external capping or targeting agents, penetrating the cell nucleus through clathrin-mediated endocytosis in less than 30 minutes. Considering that very few nuclear labelling commercial dyes are available for cellular imaging, TTA-DFP CONs are a novel and cost-effective alternative. Research on further biomedical applications such as targeted delivery of drugs to the nucleus is ongoing.

\section{Conflicts of interest}

There are no conflicts to declare.

\section{Acknowledgements}

The research described here was sponsored by New York University Abu Dhabi (NYUAD), UAE, and we are thankful for its generous support of the research program. The research was carried out using the Core Technology Platform resources at NYUAD. We would like to acknowledge the Al Jalila Foundation (AJF 201646). The authors also thank Miss Khulood Alawadi for 
3D cartoons. F. G. acknowledges the Spanish Ministry of Economy and Competitiveness for funding through the "Ramón y Cajal" and "Retos de la Sociedad (CTQ2017-87262-R)" programs.

\section{References}

1 P. J. Waller, F. Gándara and O. M. Yaghi, Acc. Chem. Res., 2015, 48, 3053-3063.

2 S. Mitra, H. S. Sasmal, T. Kundu, S. Kandambeth, K. Illath, D. Díaz Díaz and R. Banerjee, J. Am. Chem. Soc., 2017, 139, 4513-4520.

3 Y. Peng, Y. Huang, Y. Zhu, B. Chen, L. Wang, Z. Lai, Z. Zhang, M. Zhao, C. Tan, N. Yang, F. Shao, Y. Han and H. Zhang, J. Am. Chem. Soc., 2017, 139, 8698-8704.

4 J. Dong, K. Zhang, X. Li, Y. Qian, H. Zhu, D. Yuan, Q.-H. Xu, J. Jiang and D. Zhao, Nat. Commun., 2017, 8, 1142.

5 G. Das, B. P. Biswal, S. Kandambeth, V. Venkatesh, G. Kaur, M. Addicoat, T. Heine, S. Verma and R. Banerjee, Chem. Sci., 2015, 6, 3931-3939.

6 A. Mal, R. K. Mishra, V. K. Praveen, M. A. Khayum, R. Banerjee and A. Ajayaghosh, Angew. Chem., Int. Ed., 2018, 57, 8443-8447.

7 H. Wang, W. Zhu, L. Feng, Q. Chen, Y. Chao, Z. Dong and Z. Liu, Nano Res., 2018, 11, 3244-3257.

8 H. Wang, W. Zhu, J. Liu, Z. Dong and Z. Liu, ACS Appl. Mater. Interfaces, 2018, 10, 14475-14482.

9 H. Soo Choi, W. Liu, P. Misra, E. Tanaka, J. P. Zimmer, B. Itty Ipe, M. G. Bawendi and J. V. Frangioni, Nat. Biotechnol., 2007, 25, 1165.

10 X. Zhang, X. Xie, H. Wang, J. Zhang, B. Pan and Y. Xie, J. Am. Chem. Soc., 2013, 135, 18-21.

11 M. K. Kumawat, M. Thakur, R. B. Gurung and R. Srivastava, Sci. Rep., 2017, 7, 15858.

12 G. Yang, C. Zhu, D. Du, J. Zhu and Y. Lin, Nanoscale, 2015, 7, 14217-14231.

13 W. Congyu, W. Chong, H. Ting, Z. Xuejiao, G. Shouwu and Z. Jingyan, Adv. Healthcare Mater., 2013, 2, 1613-1619.

14 R. Liu, D. Wu, X. Feng and K. Müllen, J. Am. Chem. Soc., 2011, 133, 15221-15223.

15 V. S. Vyas, M. Vishwakarma, I. Moudrakovski, F. Haase, G. Savasci, C. Ochsenfeld, J. P. Spatz and B. V. Lotsch, Adv. Mater., 2016, 28, 8749-8754.

16 P. Ding, H. Wang, B. Song, X. Ji, Y. Su and Y. He, Anal. Chem., 2017, 89, 7861-7868.

17 P. Kuhn, M. Antonietti and A. Thomas, Angew. Chem., Int. Ed., 2008, 47, 3450-3453.

18 J. Guo, Y. Xu, S. Jin, L. Chen, T. Kaji, Y. Honsho, M. A. Addicoat, J. Kim, A. Saeki, H. Ihee, S. Seki, S. Irle, M. Hiramoto, J. Gao and D. Jiang, Nat. Commun., 2013, 4, 2736.

19 M. J. Bojdys, S. A. Wohlgemuth, A. Thomas and M. Antonietti, Macromolecules, 2010, 43, 6639-6645.
20 M. J. Bojdys, J. Jeromenok, A. Thomas and M. Antonietti, Adv. Mater., 2010, 22, 2202-2205.

21 S. Dalapati, E. Jin, M. Addicoat, T. Heine and D. Jiang, J. Am. Chem. Soc., 2016, 138, 5797-5800.

22 S. K. Nune, P. Gunda, P. K. Thallapally, Y.-Y. Lin, M. Laird Forrest and C. J. Berkland, Expert Opin. Drug Delivery, 2009, 6, 1175-1194.

23 J. Shen, Y. He, J. Wu, C. Gao, K. Keyshar, X. Zhang, Y. Yang, M. Ye, R. Vajtai, J. Lou and P. M. Ajayan, Nano Lett., 2015, 15, 5449-5454.

24 K. R. Paton, E. Varrla, C. Backes, R. J. Smith, U. Khan, A. O'Neill, C. Boland, M. Lotya, O. M. Istrate, P. King, T. Higgins, S. Barwich, P. May, P. Puczkarski, I. Ahmed, M. Moebius, H. Pettersson, E. Long, J. Coelho, S. E. O'Brien, E. K. McGuire, B. M. Sanchez, G. S. Duesberg, N. McEvoy, T. J. Pennycook, C. Downing, A. Crossley, V. Nicolosi and J. N. Coleman, Nat. Mater., 2014, 13, 624 .

25 C. Backes, N. C. Berner, X. Chen, P. Lafargue, P. LaPlace, M. Freeley, G. S. Duesberg, J. N. Coleman and A. R. McDonald, Angew. Chem., 2015, 127, 2676-2680.

26 Y. Hernandez, V. Nicolosi, M. Lotya, F. M. Blighe, Z. Sun, S. De, I. T. McGovern, B. Holland, M. Byrne, Y. K. Gun'Ko, J. J. Boland, P. Niraj, G. Duesberg, S. Krishnamurthy, R. Goodhue, J. Hutchison, V. Scardaci, A. C. Ferrari and J. N. Coleman, Nat. Nanotechnol., 2008, 3, 563.

27 S. D. Bergin, Z. Sun, D. Rickard, P. V. Streich, J. P. Hamilton and J. N. Coleman, ACS Nano, 2009, 3, 2340-2350.

28 S. Mitra, S. Kandambeth, B. P. Biswal, A. Khayum M, C. K. Choudhury, M. Mehta, G. Kaur, S. Banerjee, A. Prabhune, S. Verma, S. Roy, U. K. Kharul and R. Banerjee, J. Am. Chem. Soc., 2016, 138, 2823-2828.

29 G. Tai, T. Hu, Y. Zhou, X. Wang, J. Kong, T. Zeng, Y. You and Q. Wang, Angew. Chem., Int. Ed., 2015, 54, 15473-15477.

30 X. Li, H. Ren, Z. Zou, J. Sun, J. Wang and Z. Liu, Chem. Commun., 2016, 52, 453-456.

31 J. Dong, X. Li, K. Zhang, Y. Di Yuan, Y. Wang, L. Zhai, G. Liu, D. Yuan, J. Jiang and D. Zhao, J. Am. Chem. Soc., 2018, 140, 4035-4046.

32 T. Levine and C. Rabouille, Curr. Opin. Cell Biol., 2005, 17, 362-368.

33 K. Bin, C. Shuquan, D. Yaodong, Y. Decai and C. Da, Small, 2010, 6, 2362-2366.

34 J. G. R. Elferink, Biochem. Pharmacol., 1979, 28, 965-968.

35 D. Devos, S. Dokudovskaya, F. Alber, R. Williams, B. T. Chait, A. Sali and M. P. Rout, PLoS Biol., 2004, 2, e380.

36 R. B. Weisenfeld, Bioorganic Med. Chem., 2007, 15, 63616366.

37 M. E. Lalande, V. Ling and R. G. Miller, Proc. Natl. Acad. Sci. U. S. A., 1981, 78, 363-367. 